\title{
Excitation of low-frequency QPOs in black hole accretion
}

\author{
D. Giannios ${ }^{1,2,3}$ and H. C. Spruit ${ }^{1}$ \\ 1 Max Planck Institute for Astrophysics, Box 1317, 85741 Garching, Germany \\ e-mail: dgiannios@physics.uoc.gr \\ 2 University of Crete, Physics Department, PO Box 2208, 71003 Heraklion, Crete, Greece \\ ${ }^{3}$ Foundation for Research and Technology-Hellas, 71110 Heraklion, Crete, Greece
}

Received 16 July 2003 / Accepted 2 July 2004

\begin{abstract}
We study possible mechanisms of excitation of quasiperiodic oscillations in the accretion flow of black hole accreters in their hard spectral states, in the context of the "truncated disk" model. Quasi-spherical oscillations of the inner ion-supported accretion flow (ISAF) can be excited by the interaction of this hot flow with the cool disk extending outward from it. The fundamental mode of (p-mode) oscillation is most easily excited, and has a frequency near the Kepler frequency at the inner edge of the cool disk. The strongest excitation mechanism is a feedback loop involving cooling of the ISAF by soft photons from the cool disk and heating of the cool disk by the ISAF, while synchrotron emission can be a relatively strong damping effect. Growth times are computed by detailed Comptonization calculations of the interaction of an idealized ISAF with a cool disk. Typical growth times as short as a few dynamical times are found, while amplitudes can be as large as $10 \%$.
\end{abstract}

Key words. accretion, accretion disks - black hole physics - radiation mechanisms: non-thermal - X-rays: stars

\section{Introduction}

A common feature of the X-ray light curves of black hole binaries (most of them transient X-ray sources) is quasiperiodic oscillations (QPOs) (narrow power peaks with high quality factor $Q \gtrsim 2$, i.e. Belloni et al. 2002). QPOs can be categorized according to their peak frequencies - as high-frequency QPOs (HFQPOs) and low-frequency QPOs (LFQPOs). HFQPOs appear at $v \gtrsim 60 \mathrm{~Hz}$ and have low rms amplitudes (typically $1 \%$ of the mean count rate). They are typically observed when black hole binaries are in the very high state, i.e. when they exhibit both a thermal component at $k_{\mathrm{B}} T \sim 1 \mathrm{keV}$ and a hard X-ray power law with photon energy index $\left(\mathrm{d} N / \mathrm{d} E \propto E^{-\Gamma}\right) \Gamma \sim 2.5$. Because of their high frequencies, they are thought to convey information about physical properties that occur near the black hole horizon. A model which explains the 2:3 frequency ratio often seen in HFQPOs is given in Rezzolla et al. (2003).

The more common LFQPOs have a characteristic frequency range of $0.1-10 \mathrm{~Hz}$, contribute significantly to the total variability (rms amplitudes of the order $10 \%$ and higher have been seen in XTE J1550-564 and XTE J1118+480) and have been observed in both the low/hard and very high spectral states (for a review see Swank 2001). Their amplitude is generally higher at higher photon energies, though exceptions occur (for example in a QPO seen in XTE J1550-564, Kalemci et al. 2001; and in Cygnus X-1, Rutledge et al. 1999). Characteristic for the low frequency QPOs is a decrease of the frequency during the decay of the transient, suggesting a physical relation between frequency and accretion rate.
In recent years, it has become increasingly clear that the hard state power density spectrum (PDS) of Cygnus X-1 and other black hole binaries shows several breaks, i.e. frequencies where the PDS steepens (Nowak et al. 1999) and that this complex structure can be described with multiple broad noise components (Nowak 2000; Nowak et al. 2002; Pottschmidt et al. 2003). Moreover, there is evidence of a correlation between the horizontal branch oscillations and lower $\mathrm{kHz}$ QPOs in $\mathrm{Z}$ and atoll sources that can be extrapolated, by more than two orders of magnitude in frequency, to include a correlation between LFQPOs and noise components in black hole binaries (Psaltis et al. 1999). In this work, we develop a model that attempts to clarify the origin of at least some of the LFQPOs.

\subsection{QPOs as diagnostics of the accretion flow}

Apart from their intrinsic interest, the oscillations are a potentially important diagnostic of the still poorly understood inner regions of the accretion flow. In the "disk corona" model (e.g. Poutanen \& Fabian 1999), a cool ( $<1 \mathrm{keV})$ disk extends to the innermost stable orbit around the hole, while the hard X-rays are produced in a magnetically heated corona above it. In the "truncated disk" model, on the other hand, the cool disk extends inward only to a transition radius $R_{\mathrm{tr}}$, inside of which the accretion takes place as an ion supported flow (Rees et al. 1982). In such flows the ion temperature is near the virial temperature, while the electrons producing the hard X-rays have a temperature around $100 \mathrm{keV}$ (Shapiro et al. 1976; Ichimaru 1977). Their hydrodynamical properties have been studied by 
Gilham (1981) and in detail by Narayan and Yi (1994, and references therein) who called them ADAFs. To distinguish them from the radiatively supported accretion flows (also called ADAF), we use here the name "ion supported accretion flow" (ISAF).

The arguments for and against either of these geometries are part of a current debate in the literature. The main obstacle to acceptance of the truncated disk model is the difficulty in visualizing a process that can make a cool disk flow to change into a hot ion supported flow, across a highly unstable region of intermediate temperatures. Without going into details, we note here that this difficulty has been overcome by Spruit \& Deufel (2002). It is shown there that viscous heating of the ions in the interaction region between the ISAF and the cool disk near the transition radius causes the cool disk to evaporate and feed the ISAF even when the transition radius is close to the last stable orbit.

Distinguishing the two accretion geometries observationally is not possible from the X-ray spectrum alone. The hope is that a prominent feature like the low-frequency QPO can help in settling the question of the accretion geometry. In the truncated disk model, the truncation radius provides a characteristic length scale and an (orbital) frequency scale that can be associated with the QPO. The dependence of frequency on accretion rate would then imply an increase of the transition radius with decreasing accretion rate. Supporting evidence for such a physical relation is found in the correlation between reflection indicators (Fe line strength and amplitude of the Compton reflection hump) and the strength of the soft component in the X-ray spectrum with QPO frequency (Zdziarski et al. 1999; Gilfanov et al. 2000).

\subsection{The importance of excitation}

The number of possible modes of oscillation in an accretion disk is as large as those of a star. Discrete spectra of trapped oscillations can occur near the last stable orbit around a hole because of the shape of the relativistic potential (Kato 1990; Honma et al. 1992; Ipser 1996; Nowak \& Wagoner 1991, 1992). But even a disk in a Newtonian potential supports trapped oscillations near its inner or outer edge (Drury 1980; Blaes \& Hawley 1988; Kaisig 1989). The spectra of such modes are quite complex (e.g. Narayan et al. 1987). It is not surprising, therefore, that picking modes to match an observation is not too difficult (e.g. Titarchuk et al. 1998).

To make progress in interpreting QPOs, criteria are needed to identify the most plausible modes. An important clue is the amplitude of the oscillation. To explain modulations in the total $\mathrm{X}$-ray luminosity as high as $10 \%$ with a hydrodynamic oscillation, the amplitude of the oscillation must be significant, not only at the larger distances where the frequency of the oscillation is presumably set, but also in the regions close to the hole where most of the X-rays are produced. Secondly, the observed flux is an average over the accretion flow, so that all nonaxisymmetric modes average out (except for strong nonlinearity, relativistic beaming, or optical depth effects). Similarly, the visibility of modes with one or more nodes in the radial part of their structure will be strongly suppressed. The most likely modes capable of producing large amplitudes in the X-ray flux are therefore axisymmetric modes with few nodes in the radial and vertical directions.

Finally, there must be a plausible mechanism to excite the mode. While broad band noise might be explainable as some consequence of turbulence in the flow (Lyubarskii 1997; Churazov et al. 2001), the high amplitudes seen in LFQPOs probably require an excitation process intrinsic to the oscillation itself.

The wide and possibly continuous variation of the LFQPO with (inferred) accretion rate is natural in the truncated disk model. In addition, the presence of two components in the accretion flow around the transition radius gives rise to possible excitation mechanisms that are absent in disk+corona models.

\section{Excitation by interaction}

An ion supported accretion flow and a cool disk partially embedded in it interact through exchange of mass, energy and angular momentum. Exchange of mass takes place in the form of an evaporation process from some part of the cool disk, as needed to produce the ISAF in the first place, and possible condensation onto the disk at other locations. The evaporation can take place at a large distance where the virial temperature is of the order $10^{6}-10^{8} \mathrm{~K}$, through processes similar to those maintaining the mass balance in the solar corona (Meyer et al. 2000; Meyer-Hofmeister \& Meyer 2001; Liu et al. 2002). The observations mentioned above, however, show that the cool disk is truncated at a much closer distance to the hole, at least in some cases. The mechanism that allows the disk to evaporate at such distances has been identified by Spruit \& Deufel (2002).

Thus assuming that the cool disk evaporates from a region near its inner edge, the resulting ISAF spreads both inward, feeding the hole and outward, spreading over the cool disk. At some distance from the inner edge, the spreading ISAF may condense back onto the cool disk, so that a region of coexistence between the cool disk and the ISAF is maintained.

An oscillation might be excited by the exchange of mass between the cool disk and the ISAF because the accretion time scale in the disk is much longer than the viscous spreading time of the ISAF. Mass evaporating from the truncation radius $R_{\mathrm{tr}}$ depletes the surface density there. When this mass condenses from the ISAF again on to the disk at some distance $R_{\text {out }}>R_{\mathrm{tr}}$, it creates an enhancement there which takes a viscous time $\left(R_{\text {out }}-R_{\mathrm{tr}}\right)^{2} / v$ to arrive at $R_{\mathrm{tr}}$. Spreading in the ISAF and condensation at $R_{\text {out }}$ can then feed the next circle.

A second possibility for excitation is through interaction by angular momentum. A known example is viscously excited oscillation found by Kato (1978), and studied in detail by Honma et al. (1992); Papaloizou \& Stanley (1986) and others. In this mechanism, a sound wave oscillation is excited viscously in a rotating flow. The amplitude of the oscillation peaks at the outer edge of the flow (Kley et al. 1993). The boundary between the ISAF and the cool disk might be sufficient to excite a sound wave in the ISAF through this mechanism.

Finally, exchange of energy between the ISAF and the cool disk can excite oscillations. The flow is cooled by soft photons 
(either internally generated by synchrotron emission or externally from the cool disk) and heated by viscous dissipation. Changes in the soft photon input heat or cool the ISAF, causing it to expand and contract on a sound crossing time scale. Since the ISAF is partially pressure supported, the relative changes in size are of the same order as the relative changes in heating or cooling rate. The size of the region of overlap with the cool disk thus varies as well, and thereby the soft photon input into the ISAF.

In the following we investigate the conditions under which interaction by exchange of energy can produce self-excited oscillations. More specifically, we focus on the variation of radiative cooling of the flow, and assume that the viscous heating does not vary. The viscous heating can excite/damp oscillations on the viscous time scale. However, we show in Sect. 5 that radiative cooling excites/damps oscillations on much shorter time scales, validating our assumption (for further discussion see Sect. 5.3).

\section{Model}

We consider low order oscillation modes of the ISAF, excited by the energetic interaction between the ISAF and the cool disk. There are different interaction paths, and whether these excite or damp an oscillation of the ISAF depends on geometrical properties and on the source of soft photons.

\subsection{Geometry and soft photon sources}

The basic ingredients of the model are a standard thin disk (Shakura \& Sunyaev 1973) truncated at a radius $R_{\mathrm{tr}}$ and an ISAF extending to a radius $R_{\text {out }}>R_{\mathrm{tr}}$. Inverse Compton scattering of soft photons is the natural radiative process to explain the non-thermal X-ray spectrum in the low/hard state, but there are several possible sources for the soft photons, depending on the geometry of the flow and model assumptions.

Soft photons can be generated internally in the ISAF as thermal synchrotron photons. This will be the dominant source of soft photons in an ion supported flow near a black hole if the magnetic field strength is some fraction of the equipartition field. This synchrotron radiation is strongly self absorbed below a characteristic turn-over frequency $v_{t}$. Most of the synchrotron flux is emitted near this frequency, which typically lies in the infra-red or the optical region of the electromagnetic spectrum.

A second source of soft photons is the radiative interaction between the hot flow and the cool disk. Hard X-rays from the inner hot flow are partly absorbed and partly reflected by the cool disk. The absorbed energy is reprocessed into thermal soft flux and emitted by the disk. These soft photons are the seed photons Comptonized by the ISAF. The energetics and spectra produced in this radiation-mediated interaction have been studied by Haardt \& Maraschi (1993).

In addition to this purely radiative interaction, the hot flow also heats the cool disk by "proton illumination" (Deufel et al. 2001; Deufel et al. 2002; Spruit \& Deufel 2002). In this process, part of the ISAF condenses onto the disk in the overlap region. Energetic protons (which carry most of the ISAF's energy) are slowed down by the disk plasma and their energy gets thermalized and appears as soft flux. The proton energy flux $F_{\mathrm{p}}$ which is released in the disk is of the order

$F_{\mathrm{p}}=n_{\mathrm{p}}\left(k_{\mathrm{B}} T_{\mathrm{p}}\right) c_{\mathrm{s}}$

where $c_{\mathrm{s}}$ is the proton sound speed and $n_{\mathrm{p}}$ is the proton number density in the ISAF. The density is of the order $n_{\mathrm{p}}=$ $\tau /\left(\sigma_{T} R_{\text {out }}\right)$, where $\tau$ is the Thomson optical depth of the ISAF and $\sigma_{T}$ the Thomson cross section.

If half of this flux is re-emitted isotropically as soft photons, the disk surface temperature $T_{\text {disk }}$ is given by the relation $F_{\mathrm{p}} / 2=\sigma T_{\text {disk }}^{4}$, with $\sigma$ the Stefan-Boltzmann constant $\left(\sigma=5.67 \times 10^{-5} \mathrm{erg} \mathrm{cm}^{-2} \mathrm{~s}^{-1} \mathrm{~K}^{-4}\right)$. We get

$T_{\text {disk }} \simeq\left(\frac{\tau}{m_{1} r^{5 / 2}}\right)^{1 / 4} \mathrm{keV}$

where $m_{1}$ is the mass of the black hole in units of 10 solar masses $\left(2 \times 10^{34} \mathrm{~g}\right)$ and $r$ the distance from the black hole in units of the Schwarzschild radius $R_{\mathrm{S}}=2 G M / c^{2}$. We see that $T_{\text {disk }} \sim 100 \mathrm{eV}$ is a typical value for $\tau$ of order unity and $r \sim 50$. In the calculations reported below, the effects of each of these sources of soft photons on the oscillation are investigated.

\subsection{The oscillations, model approximations}

The excitation mechanisms considered here operate on the balance between heating and cooling in the ISAF, in particular through a modulation of the soft photon input. The simplest modes and the most likely ones to be excited by this mechanism are the axisymmetric modes with few nodes in the radial direction, in particular the fundamental mode. In the following, we restrict ourselves to the fundamental mode of the ISAF. As in a star, it has a frequency of the order of the Kepler frequency at its outer edge, or the inverse time for a sound wave to travel across it.

Since the fundamental mode senses only the average sound speed, it is sufficient to model the ISAF as spherical. Since the rotation period is of the order of the sound crossing time, its effects on the fundamental mode are modest, hence we also ignore the effect of rotation on the mode. Each of these approximations can be easily improved on with a more detailed model of the structure of an ISAF. At the present level of understanding of the geometry of the accretion flow onto black holes, this is not a primary concern.

We further simplify the model by taking the equilibrium electron temperature $T_{\mathrm{e}}$ and density $\rho_{0}$ as homogeneous. The fundamental mode of oscillation is approximated by a homologous expansion and contraction.

Thus, if $R\left(t, R_{0}\right)$ is the radial position of a shell of mass with equilibrium position $R_{0}$, a sinusoidal oscillation of the model ISAF is described by

$R\left(t, R_{0}\right)=R_{0}[1+a \cdot \sin (\omega t)]$,

where $a$ is the fractional amplitude of the oscillation. The radial velocity and the density perturbation are

$v\left(t, R_{0}\right)=a \omega R_{0} \cos (\omega t), \quad \Delta \rho=-3 a \rho_{0} \sin (\omega t)$. 
Also the perturbation of the optical depth of the ISAF $(\tau=$ $\left.\rho_{0} \kappa_{\mathrm{es}} R_{\text {out }}\right)$ is

$\Delta \tau=-2 a \tau \sin (\omega t)$

Such a non-rotating, constant density configuration in homologous motion has a fundamental frequency of oscillation (Cox 1980)

$\omega=\sqrt{(3 \gamma-4) \frac{G M}{R_{\mathrm{out}}^{3}}}$,

where $\gamma$ is the ratio of specific heats, assumed constant. For the ion cloud of an ISAF, the non-relativistic value $\gamma=5 / 3$ is a good approximation, which will be used in this work. The oscillation frequency is then exactly equal to the Kepler frequency $\omega_{k}$. (In view of the approximations made, this exact agreement is a coincidence.)

\subsection{Excitation and damping}

An efficient excitation mechanism is needed, since the observed oscillation is often quite strong, accounting for a large fraction of rms variability. In the present model, the excitation is due imbalance of cooling and heating terms during the oscillation, such that the net work done on the gas by the pressure forces is positive during an oscillation cycle.

With standard stellar oscillation theory (see for example, Cox 1980; Unno et al. 1979), the rate of excitation of an oscillation can be expressed by the work integral

$\langle\dot{W}\rangle=-\frac{1}{T} \int_{m} \mathrm{~d} m \int_{0}^{T}(\gamma-1) \frac{\Delta \rho}{\rho} \Delta \dot{q} \mathrm{~d} t$.

Where $\Delta \dot{q}$ is the (Lagrangian) variation of the net cooling rate per unit mass during the oscillation, the first integral is over the mass of the ISAF and the second over the oscillatory period $T$. An ideal gas with constant ratio of specific heats $\gamma$ has again been assumed.

If the excitation rate is small compared with the oscillation frequency (as needed in order for the quality factor $Q$ of the oscillation to be high), the work function can be represented by difference in the net cooling rate at the maxima and minima of the oscillation (see Appendix A)

$\langle\dot{W}\rangle=3(\gamma-1) a \frac{\dot{q}_{\exp }-\dot{q}_{\mathrm{contr}}}{4}$,

where $a$ is the relative displacement amplitude as before, and $\dot{q}_{\text {exp }}, \dot{q}_{\text {contr }}$ are the net cooling rates at maximum expansion and maximum contraction, respectively.

If cooling mechanisms are more efficient in the expanded phase of the oscillation than in the contracted phase, then in every oscillatory cycle there is a net increase of the oscillation's energy proportional to $\dot{q}_{\text {exp }}-\dot{q}_{\text {contr }}$ which means excitation of the oscillation. If, on the other hand, $\dot{q}_{\exp }-\dot{q}_{\text {contr }}<0$ then the oscillation gradually decays.

\subsection{Effect of different sources of soft photons}

To find out whether a mode can be excited by the processes that determine the energy balance, special attention must be paid to the cooling mechanisms of the ISAF, since these are more easily modified than the viscous heating of the flow (cf. Sect. 5.3). Inverse Compton accounts for most of the cooling of the ISAF (at least for the conditions under which the observed spectra are produced, since these are dominated by hard X-rays). Soft flux, that crosses the hot and optically thin medium of the ISAF, is upscattered by energetic electrons resulting in energy transfer from the electrons to the radiation field. The cooling rate of the ISAF is thus controlled by the input of soft photons. As discussed above, we consider three sources of soft photons:

i) synchrotron photons from the inner parts of the flow,

ii) irradiation of the thin disk by X-rays from the ISAF,

iii) proton illumination of the thin disk in the overlap area. The strength of Compton cooling is determined by the parameter, $y=4 k_{\mathrm{B}} T_{\mathrm{e}} /\left(m_{\mathrm{e}} c^{2}\right) \tau$ and the soft flux: $\dot{q}_{\mathrm{c}}=L_{\mathrm{soft}}\left(\mathrm{e}^{y}-1\right)$. During the expansion phase the optical depth of the ISAF drops (Eq. (5)) and so does the electron temperature (see next section), resulting in a decrease of the $y$ parameter. The opposite trend is observed during contraction. So, if the soft flux does not vary much, the cooling rate of the expanded phase must always be less than the cooling rate of the contracted phase. This leads to damping of the oscillations. On the other hand if the soft flux increases quickly enough during expansion, there can be excitation of the oscillations.

When synchrotron photons are the "seed" photons to be Comptonized, then it is straightforward to show that they act as a damping mechanism. This is because the synchrotron flux increases strongly with electron temperature and the strength of the magnetic field and less strongly with the Thompson optical depth (e.g. Wardziński \& Zdziarski 2000). The magnetic field scales as $B^{2} \sim P_{\text {gas }}$, which means that $B, T_{\mathrm{e}}$ and $\tau$ all decrease during expansion. The synchrotron soft flux decreases and so does the cooling rate during expansion, resulting in energy loss of the oscillation.

On the other hand, in the irradiation and proton illumination cases, it is not a priori clear whether the net effect is damping or exciting. Detailed calculations of the cooling rates are needed to settle this question.

\section{Calculations}

\subsection{The electron temperature in equilibrium}

The energy source of the electrons in the two temperature plasma is the transfer of energy from the hot protons through Coulomb interactions. This causes the protons to cool at a time scale (Spitzer 1956; Stepney 1983) $t_{\mathrm{p}}$

$t_{\mathrm{p}}=\sqrt{\frac{\pi}{2}} \frac{m_{\mathrm{p}}}{m_{\mathrm{e}}} \frac{1}{n \sigma_{T} c \ln \Lambda}\left(\frac{k_{\mathrm{B}} T_{\mathrm{e}}}{m_{\mathrm{e}} c^{2}}\right)^{3 / 2}$,

where $T_{\mathrm{e}}$ stands for the electron temperature, $n=\tau /\left(\sigma_{\mathrm{T}} R_{\mathrm{out}}\right)$ is the number density of electrons, $\ln \Lambda$ is the Coulomb logarithm. This expression is the non-relativistic one and applies when 
$T_{\mathrm{e}} \ll T_{\mathrm{p}} \ll m_{\mathrm{p}} T_{\mathrm{e}} / m_{\mathrm{e}}$. Where $T_{\mathrm{p}}$ is the proton temperature assumed to be the virial temperature

$T_{\mathrm{p}}=T_{\mathrm{vir}}=\frac{G M m_{\mathrm{p}}}{3 k_{\mathrm{B}} R} \simeq \frac{156}{r} \mathrm{MeV}$

As will be shown in the rest of the section, these inequalities hold for most of the parameter space of $T_{\mathrm{e}}, T_{\mathrm{p}}$ in which we are interested in this work.

Because of their lower temperature, the heating time scale of the electrons is shorter than the proton cooling time scale

$t_{\mathrm{e}}=\frac{T_{\mathrm{e}}}{T_{\mathrm{p}}} t_{\mathrm{p}}$.

The heating rate of the electrons (erg/s) over the whole ISAF is then given by $n k_{\mathrm{B}} T_{\mathrm{e}} V / t_{\mathrm{e}}$, with $V$ being the volume of the ISAF. In equilibrium this quantity equals the cooling rate of the electrons, which is assumed to be controlled by Compton cooling and ultimately equal to the observed X-ray flux of the source

$L_{\mathrm{x}}=\frac{n k_{\mathrm{B}} T_{\mathrm{e}} V}{t_{\mathrm{e}}}$.

Combining this equation with Eqs. (9), (11), $V=(4 / 3) \pi R_{\text {out }}^{3}$ and taking $\ln \Lambda \simeq 20, T_{\mathrm{p}}=T_{\text {vir }}$, we can relate the temperature to the optical depth and $\mathrm{X}$-ray flux

$k_{\mathrm{B}} T_{\mathrm{e}} \simeq 5 \tau^{4 / 3}\left(\frac{L_{\mathrm{E}}}{L_{\mathrm{x}}}\right)^{2 / 3} \quad \mathrm{keV}$

(Shapiro et al. 1976). Here $L_{\mathrm{E}}$ is the Eddington luminosity.

A further constraint on $T_{\mathrm{e}}$ and $\tau$ from observable quantities comes from the slope $s$ of the X-ray spectrum in a $v \cdot F_{v}$ diagram $\left(v \cdot F_{v} \sim v^{s}\right)$. With an analytic fit to Comptonized spectra given in Wardziński \& Zdziarski (2000) their Eq. (22), which agrees with reasonable accuracy with our computational results in Sect. 5.4) we get for the hard X-ray power-law slope

$s \simeq 1-\frac{0.8}{\tau^{4 / 5}} \frac{100 \mathrm{keV}}{k_{\mathrm{B}} T_{\mathrm{e}}}, \quad 0.5 \leq \tau \leq 2, \quad k_{\mathrm{B}} T_{\mathrm{e}} \leq 200 \mathrm{keV}$.

Equations (13) and (14) determine the electron temperature of the ISAF, expected from electron heating through Coulomb interaction with virial protons, in terms of the optical depth $\tau$ of the flow and two observables, the X-ray luminosity and the slope of the hard X-ray spectrum. This is shown in Fig. 1.

From Fig. 1 one can see that the electron temperature of the ISAF, if it is to account for the observations, is $\sim 100 \mathrm{keV}$. Furthermore since $T_{\mathrm{p}}=T_{\mathrm{vir}}$, the inequalities $T_{\mathrm{e}} \ll T_{\mathrm{p}} \ll$ $m_{\mathrm{p}} T_{\mathrm{e}} / m_{\mathrm{e}}$ hold for $r_{\text {out }}<1000$ validating the use of Eq. (9).

If the optical depth is expressed in terms of the accretion rate, with an assumed viscosity, this can be further reduced to a dependence of the expected electron temperature on accretion rate (e.g. Rees et al. 1982; Narayan \& Yi 1995; Zdziarski 1998).

\subsection{The electron temperature during oscillation}

In order to calculate the excitation of an oscillation, the net cooling/heating of the flow has to be followed during a cycle (Sect. 3.3). For this we need the variation of the electron

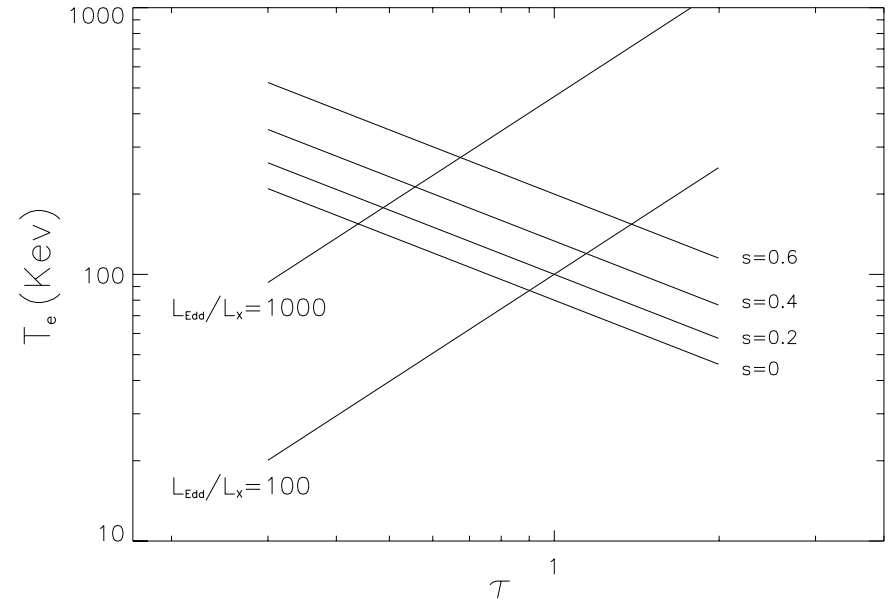

Fig. 1. Electron temperature in an ion supported flow of optical depth $\tau$, as a function of the observed X-ray luminosity $L_{\mathrm{x}}$ and luminosity index $s$ of the spectrum $\left(v F_{v} \sim v^{s}\right)$.

temperature during the oscillation. We compare the electron heating time scale with half of the oscillation period $P$ (time between two passes through the equilibrium position). From Eqs. (9)-(11) the heating time scale of the electrons is

$t_{\mathrm{e}}=6 \times 10^{-7} \frac{m_{1} r_{\mathrm{out}}^{2}}{\tau}\left(\frac{k_{\mathrm{B}} T_{\mathrm{e}}}{100 \mathrm{keV}}\right)^{5 / 2} \mathrm{~s}$,

and

$P / 2=4.3 \times 10^{-4} m_{1} r_{\text {out }}^{3 / 2} \quad \mathrm{~s}$,

where $r_{\text {out }}$ is the radius of the ISAF in $R_{\mathrm{S}}$. It follows that $t_{\mathrm{e}} \ll$ $P / 2$ for optical depths $>0.01$ and $r_{\text {out }} \lesssim 1000$.

The electron temperature therefore adjusts quasistatically to the changing proton temperature, so equilibrium equations from the last section still apply during the oscillation. Taking the Lagrangian derivative $(\Delta)$ of Eq. (11) and using $\Delta n=$ $-3 a n \sin (\omega t)$ and $\Delta T_{\mathrm{p}}=-2 T_{\mathrm{p}} \sin (\omega t)$ (see next section) we find that

$\Delta t_{\mathrm{e}}=5 t_{\mathrm{e}}\left[a \sin (\omega t)+\frac{\Delta T_{\mathrm{e}}}{2 T_{\mathrm{e}}}\right]$.

Since the adjustment time of the electron temperature is short, the heating rate of the electrons must equal the (Compton) cooling rate $\dot{q}_{\text {cool }}$ at any instant:

$\dot{q}_{\text {heat }}=\frac{n k_{\mathrm{B}} T_{\mathrm{e}} V}{t_{\mathrm{e}}}=\dot{q}_{\mathrm{cool}}$.

\subsection{Evaluation of the work integral}

The oscillations involve the pressure force and gravity. In an ion supported flow, both these forces are concentrated in the protons. Hence the cooling/heating rate $\dot{q}$ in the work integral involves only the proton temperature and the distance from the central mass

$\dot{q}=\dot{q}\left(T_{\mathrm{p}}, r(t)\right)$.

The proton temperature $T_{\mathrm{p}}$ of a mass element at distance $r(t)$ changes with time due to three factors, the compression/expansion of the gas, energy transfer to the electrons, and 
the viscous heating. Variations in viscous heating as a possible excitation mechanism of oscillations in accretion disks has been studied before (Kato 1978; Papaloizou \& Stanley 1986; Kley et al. 1993). The maximum growth rate due to this process is of order $\alpha \omega$, where $\alpha$ is the disk viscosity parameter. Assuming $\alpha$ to be small, perhaps of order $0.01-0.1$ as indicated by numerical simulations of magnetic accretion disk turbulence (e.g. Brandenburg et al. 1997; Armitage 1998), this contribution is small compared with the growth rate we find below due to the other interaction processes, hence we will neglect variations in viscous dissipation during the oscillation cycle in the following (see also Sect. 5.3).

Assuming we are looking at a slowly damped or growing mode, the temperature variation can be written as $\Delta T=\Delta T_{\mathrm{ad}}+$ $\epsilon \Delta T_{\text {cool }}$, where $\Delta T_{\text {ad }}(t)$ is the adiabatic temperature variation, $\Delta T_{\text {cool }}$ is the temperature change due to the variation in energy transferred to the electrons, and $\epsilon$ a small number. Expanding in $\epsilon$, the work integral can be evaluated by setting $\Delta T=\Delta T_{\mathrm{ad}}$ in Eq. (19), that is, it can be found to lowest order using only the adiabatic variation of the proton temperature.

In practice we evaluate the work integral in a highly discretized way: the integral is represented by the sum of two values: the integrands at the phases of maximum contraction and maximum expansion. For a linear (sinusoidal) oscillation, evaluation at a single known phase in the oscillation would be enough to determine its amplitude, and would be sufficient to determine the work integral. By evaluation at the phases of both maximum and minimum expansion, nonlinearity due to asymmetry between the expanded and contracted phases can be taken into account to lowest order. This is somewhat important for the present problem because an asymmetry is in fact expected since, for large oscillatory amplitude, the ISAF in its contracted phase can actually detach from the cool disk. For the two phases, the cooling rate is evaluated by an iterative procedure, varying the electron temperature until the balance Eq. (18) is satisfied.

\subsection{The Monte-Carlo code}

In order to study both the excitation and the damping mechanisms that take place in the ISAF quantitatively, the Comptonization of soft radiation has to be calculated in detail. For this we have performed Monte Carlo simulations following Pozdnyakov et al. (1983). For specific values of the electron temperature $T_{\mathrm{e}}$, the optical depth $\tau$, location and energy of the soft photons, we calculate the cooling rate of the ISAF as well as the X-ray spectrum produced.

The location of the soft photons depends on the mechanism that produces them. In the case of thermal synchrotron photons, they are expected to be emitted in the region close to the black hole, with an energy distribution that strongly peaks at a few eV. For simplicity we assume that all synchrotron photons come from the center of the ISAF. If the soft photon flux is a result of disk irradiation caused by hard X-rays coming from the inner hot flow, then the location of their emission and their energy is calculated by the iterative method that is described in Sect. 5.1. Finally in the "proton illumination" case, the soft flux comes from the inner part of the thin disk where the disk interacts with the ISAF (at radii $R_{\mathrm{tr}}<r<R_{\text {out }}$ ). The photon energy in this case is given by Eq. (2).

The Monte Carlo method follows the path of a large number of photons and makes a statistical description of the radiation transfer inside the scattering medium. The calculation takes properly into account the quantum mechanical and special relativistic effects of the microphysics of the scattering. We make sure that the statistical sample (i.e. the number of photons) is large enough to keep statistical noise at a low level.

\section{Results}

Whether small amplitude radial oscillations of the ISAF, once appearing, will increase in amplitude or die out depends on the origin of the soft photons. As discussed in Sect. 3.1, there are three possible sources of soft photons: the reprocessing of hard (inverse Compton) photons intercepted by the cool disk (the "X-ray irradiation" model), photons produced by ion-heating of the cool disk ("proton illumination"), and internal (synchrotron) photons generated in the ISAF. The conditions for excitation are different in each case. Although all these processes may contribute, we study their effects separately. We start with the irradiation model.

\subsection{X-ray irradiated cool disk}

Parameters of the ISAF are its equilibrium values of electron temperature and optical depth, using Fig. 1 as a guide. The degree of overlap of the ISAF over the cool disk in the equilibrium state, $f=R_{\text {out }} / R_{\text {tr }}-1$, is a third parameter of the problem. We make a guess of the initial radial temperature profile of the disk surface and simulate the emission of cool photons from the disk and the upscattering of these soft photons into the ISAF with a Monte Carlo calculation. Part of the hard X-rays produced by inverse Compton scattering in the ISAF will meet the thin disk in their scattering random walk and get either absorbed or reflected. The absorbed X-ray flux, assumed to be thermalized into a black body, determines the new temperature profile of the disk surface. The simulation is repeated using the new temperature profile several times until it converges (within numerical uncertainties imposed by the statistical nature of the Monte Carlo method). The cooling rate of the ISAF in the final run is recorded.

The next step is to expand the medium by a small amount. The optical depth of the hot medium is reduced in accordance to Eq. (5) for $\sin (\omega t)=1$ (maximum expansion), while the temperature of the hot medium on the expanded phase is given a trial value. Following the procedure described in the previous paragraph, the temperature profile of the disk and the cooling rate of the ISAF $\dot{q}_{\text {exp }}$ are computed. Then we iterate varying the temperature of the ISAF until Eq. (18) is satisfied. The same procedure can be applied to maximum contraction of the ISAF to compute its cooling rate $\dot{q}_{\text {contr. }}$.

There are two simplifications made during this computation. First, we have ignored the reflected component. Detailed spectral predictions are out of the scope of this work and, on the other hand, the reflected spectrum lies in the X-ray regime 


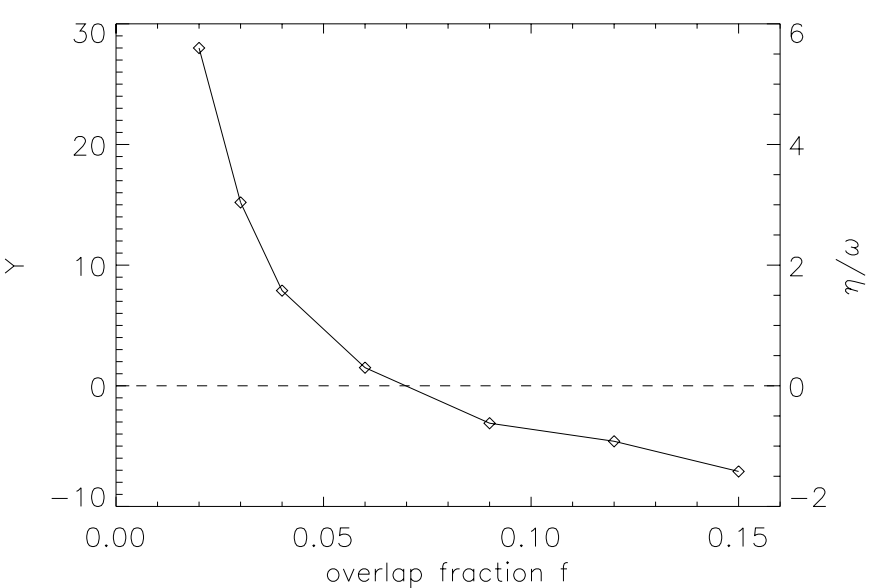

Fig. 2. The growth/damping rates of small amplitude oscillations for different overlap fractions $f=\left(R_{\text {out }}-R_{\text {tr }}\right) / R_{\text {out }}$ in the proton illumination case. The left vertical axis shows the work integral $Y=$ $\left(\dot{q}_{\text {exp }}-\dot{q}_{\text {contr }}\right) /\left(a \dot{q}_{\text {eq }}\right)$, the right axis the corresponding growth rate $\eta$ in units of the frequency $\omega$ of the oscillation. For $f<0.07$ the work integral is positive and the oscillations are excited.

and is not expected to contribute much in the ISAF cooling (in which we are mainly interested). Second, we kept the fraction of the flux that the disk reflects in the different phases of the oscillation constant. This, however, may not be the case (see for example, Nayakshin et al. 2000).

Keeping this in mind, we find that in the irradiation model $\dot{q}_{\text {exp }}-\dot{q}_{\text {contr }}<0$ for the entire range of the parameter space $\tau, T_{\mathrm{e}}, f$ that we have investigated. It seems to be a robust result that when soft photons are product of reprocessing of $X$-rays by the surface of the thin disk, small amplitude radial oscillations of the ISAF are damped.

\subsection{Proton illumination}

In the proton illumination model the soft photon flux comes from the overlap region only. Part of the proton illumination energy (about half, like in the Haardt-Maraschi energy balance) is reprocessed into blackbody radiation and the rest is emitted in the X-ray band due to the "warm" layer that forms in the overlap region (see Deufel \& Spruit 2000 for details). Here we will ignore the X-rays coming from the warm layer. The total soft luminosity is

$L_{\mathrm{soft}} \simeq \frac{1}{2} F_{P} A$,

where $F_{P}$ is given by Eq. (1) and $A=2 \pi\left(R_{\mathrm{o}}^{2}-R_{\mathrm{tr}}^{2}\right)$ is the overlap surface area (the factor 2 accounts for the two sides of the disk). Taking the Lagrangian derivative of Eq. (20), we arrive at the result

$\Delta L_{\text {soft }}=2 L_{\text {soft }}\left(\frac{3 K^{2}-2}{1-K^{2}}\right) a \sin (\omega t)$,

where $K=R_{\text {tr }} / R_{\text {out }}$, or $f=1-K$. From this equation it follows that for $K^{2}<2 / 3$ (which corresponds to $f>0.18$ ), the soft flux decreases on expansion and increases on contraction of the ISAF. So for large overlap fraction, $f>18 \%$, any small amplitude radial oscillations are expected to be damped (see
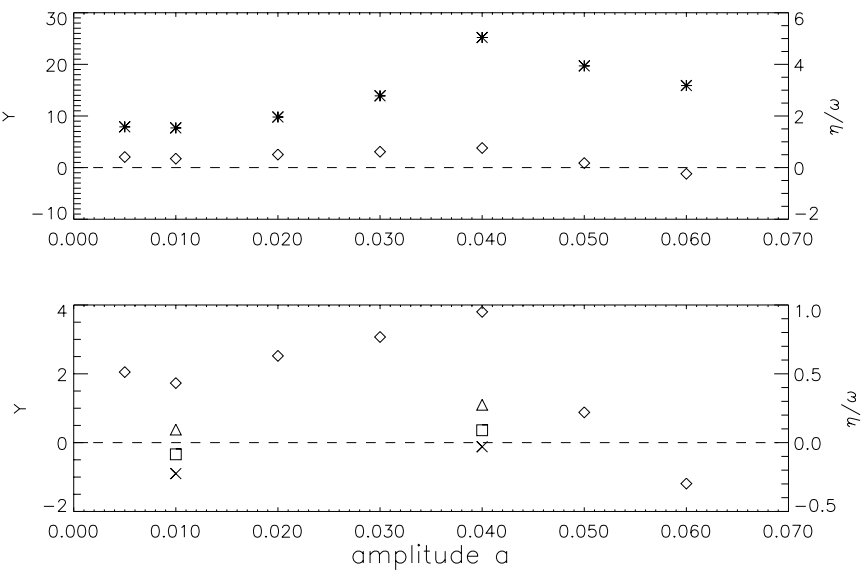

Fig. 3. Work integral $Y$ of large amplitude oscillations. Upper panel: the proton illumination case (asterisks) and the case where there is also a soft synchrotron flux with $L_{\mathrm{illum}} / L_{\mathrm{syn}}=230$ (diamonds). Lower panel: $L_{\mathrm{illum}} / L_{\mathrm{syn}}=230$ (diamonds), 163 (triangles), 150 (squares), 138 (X). Note that for $L_{\text {illum }} / L_{\text {syn }}=150$, small amplitude oscillations are damped, while larger amplitude oscillations $(a \sim 4 \%)$ are excited.

also the discussion of the the synchrotron soft photon case in Sect. 3.1). Of more interest is the regime of $f=1-R_{\text {tr }} / R_{\text {out }}<$ $10 \%$.

From calculations with different fractional overlap $f$, we find that there is a characteristic value $f_{\mathrm{o}}$ below which oscillations are excited. This is illustrated in Fig. 2. It shows, as a measure of the work integral, the quantity $Y=\left(\dot{q}_{\exp } / \dot{q}_{\text {contr }}-1\right) / a$, the fractional variation of the cooling rate, divided by the relative amplitude $a$ of the oscillation. It is proportional to the growth rate $\eta$ of the oscillations (see Appendix A). For the example shown $\left(\tau=1.5\right.$ and $\left.T_{\mathrm{e}}=130 \mathrm{keV}\right)$ the regime of excitation is $f \leq 0.07$. The results remain qualitatively the same for different choices of $T_{\mathrm{e}}$ and $\tau$.

Until now we have only dealt with small amplitude oscillations. What limits the growth of these oscillations? To answer this questions we have also calculated the variation of the cooling rate for larger amplitudes. The value of the amplitude at which the work integral changes sign is a good estimate of the amplitude reached by the oscillation. Again we approximate the work integral by the sum of the integrand in the expanded and contracted phases.

Figure 3 (upper plot) shows the results for an equilibrium overlap fraction $f=0.04$. The growth rate has a maximum for $a=0.04$. When $a>0.04$ the ISAF, during part of the contracting phase, does not interact with the disk $(f=0)$ and $\mathrm{X}$-ray flux ceases. Note that the work integral is positive for all amplitudes: there is no limit to the growth in this simplified case. More realistically, hydrodynamic nonlinearities ignored here would limit the oscillation. Also, other sources of soft photons will have a limiting effect, as discussed below.

\subsection{Combination of different soft photon sources}

The situation described in the previous section leaves out some physics. Synchrotron photons from the ISAF and soft photons produced by irradiation of the cool disk are expected to also play a role in determining the amplitude of the oscillation, since 
we have already shown that they act as damping mechanisms. As a first step in this direction, we now include synchrotron photons into the simulation. The amount of synchrotron soft photons produced by the ISAF depends on the unknown field strength. Hence we treat the synchrotron luminosity $L_{\text {syn }}$ as a free parameter. The soft flux due to proton illumination in this example is a black body with $T_{\text {illum }}=100 \mathrm{eV}$, while the typical energy of the synchrotron photons is set at $\sim 3 \mathrm{eV}$. The values of the other parameters are $T_{\mathrm{e}}=130 \mathrm{keV}, \tau=1.5, f=0.04$.

For $L_{\text {illum }} / L_{\text {syn }}=230$ (Fig. 3 ), the oscillation amplitude grows up to $a \sim 0.05$ and stabilizes at that level. A weak synchrotron flux can limit the amplitude's growth to values slightly larger than the overlap fraction. However, if the synchrotron flux is strong enough, $L_{\text {illum }} / L_{\text {syn }} \lesssim 140$, the oscillation does not get excited at all.

An interesting feature of the combined synchrotron-proton illumination model for the soft photons is the appearance, in this example, of "hard" oscillations. As Fig. 3 shows, small amplitude oscillations are damped, while the work integral is positive for oscillations with larger amplitudes, $a \sim 0.04$, if the synchrotron flux is in the range $140 \lesssim L_{\text {illum }} / L_{\text {syn }} \lesssim 155$.

In this work we have ignored any variation of the viscous heating during the oscillation. However, viscous heating can trigger/damp oscillations on the viscous time scale (e.g. Kley et al. 1993). Following the $\alpha$ viscosity prescription (Shakura \& Sunyaev 1973) and taking that $H \simeq R$ for an ISAF, we have $t_{\text {visc }} \sim t_{\text {dyn }} / \alpha$, where $H$ is the thickness of the ISAF and $t_{\text {dyn }}=1 / \omega$ is the dynamical time scale. So the viscous excitation/damping rate is $\eta_{\text {visc }} / \omega \sim \alpha$. Comparing this with the rates due to radiative cooling (Figs. 2, 3), we see that for $\alpha \lesssim 0.1$ the effects of viscous heating on excitation can be safely neglected.

\subsubsection{Reprocessed hard X-rays}

We now consider the case when the soft photon flux is produced by the joint effects of proton illumination and reprocessing of the hard X-rays from the ISAF. Keeping the proton illumination component of the soft flux as a black body with $T_{\text {illum }}=100 \mathrm{eV}$ and, calculating the temperature profile of the disk due to X-ray irradiation with the method described in Sect. 5.1, we study whether small amplitude oscillations are excited or damped for different overlap fractions $f$. In this example we have fixed $k_{\mathrm{B}} T_{\mathrm{e}}=150 \mathrm{keV}$ and $\tau=1$.

The results are presented in Fig. 4, showing the growth rate as a function of the overlap fraction and oscillation amplitude. For $f \lesssim 0.05$ small amplitude oscillations are excited. Fixing the equilibrium overlap fraction $f$ to 0.04 , we have also calculated the variation of the cooling rate of the ISAF for larger oscillation amplitudes (lower panel). The maximum growth rate occurs at an amplitude $a=0.04$. The amplitude of the oscillation can be estimated from the change of sign of the grwoth rate as a function of amplitude, yielding $\alpha_{\max } \sim 0.09$.

\subsubsection{Viscous dissipation in the cold disk as a source of soft photons}

A source of soft photons ignored so far is viscous dissipation in the thin disk. Including this soft-photon source, using standard
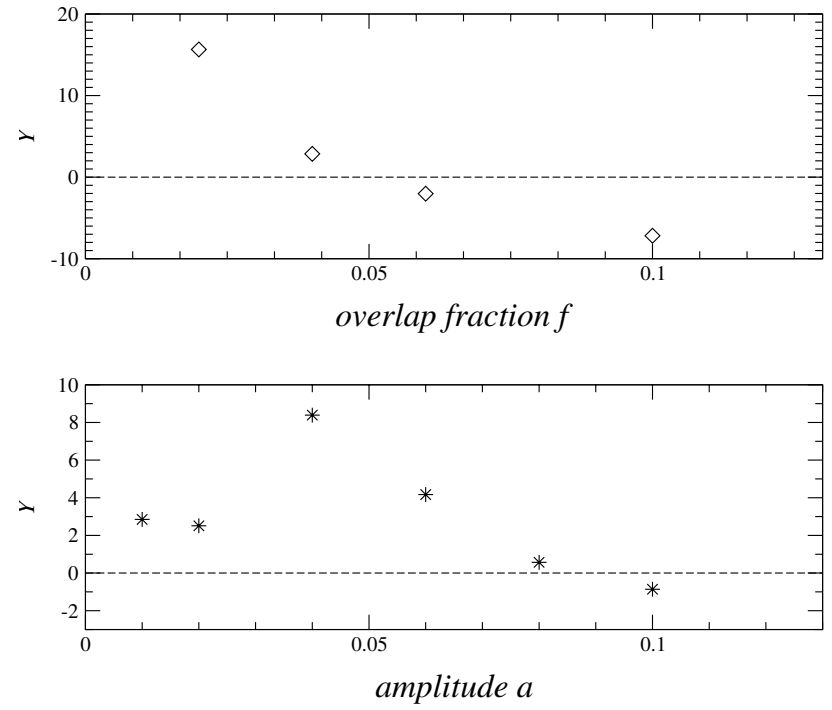

Fig. 4. Work integral for the case where both proton illumination and irradiation are taken into account. Upper panel: small amplitude oscillations as a function of overlap fraction $f$. For $f \lesssim 0.05$ small amplitude oscillations are excited. Lower panel: work integral as a function of oscillation amplitude, for fixed overlap fraction $f=0.04$. In this case the oscillation grows up to $a \sim 0.09$. These results are for ISAF temperature $k_{\mathrm{B}} T_{\mathrm{e}}=150 \mathrm{keV}$, and $\tau=1$.

thin disk expressions (Shakura \& Sunyaev 1973), we find that the results presented in the previous sections remain essentially the same. This is so because the intrinsic luminosity of the disk has only weak triggering/damping effects on the oscillations of the ISAF.

\subsection{Spectral evolution during the oscillation}

From the discussion in the previous sections, it is evident that if the overlap fraction between the thin disk and the ISAF is small enough (typically smaller than 7\%), radial oscillations of the ISAF can be excited. The overlap region is a source of hard X-rays (Deufel et al. 2001, 2002). The ISAF itself, however, may actually be the main X-ray producing region, and its oscillations are expected to have direct implications on the shape of the emitted spectrum.

On expansion of the ISAF the optical depth, the electron temperature and hence the Compton $y$ parameter drop. The soft photon input on the other hand increases. As a result the spectrum becomes softer while the total X-ray flux increases.

The opposite happens in the contracting phase when the source becomes harder and the X-ray flux decreases. So the $\mathrm{X}$-ray spectrum exhibits a characteristic pivoting behavior which is shown in Fig. 5. In this figure (which corresponds to the case where both proton illumination and synchrotron photons are taken into account) we have plotted the spectrum computed by the Monte Carlo code in equilibrium position, in maximum expansion and in maximum contraction.

One clear conclusion from Fig. 5 is that the oscillations are predicted to be soft i.e. to have more power in soft X-rays than in hard X-rays. A second conclusion is that a simultaneous oscillation (but of opposite phase) should be observed at 


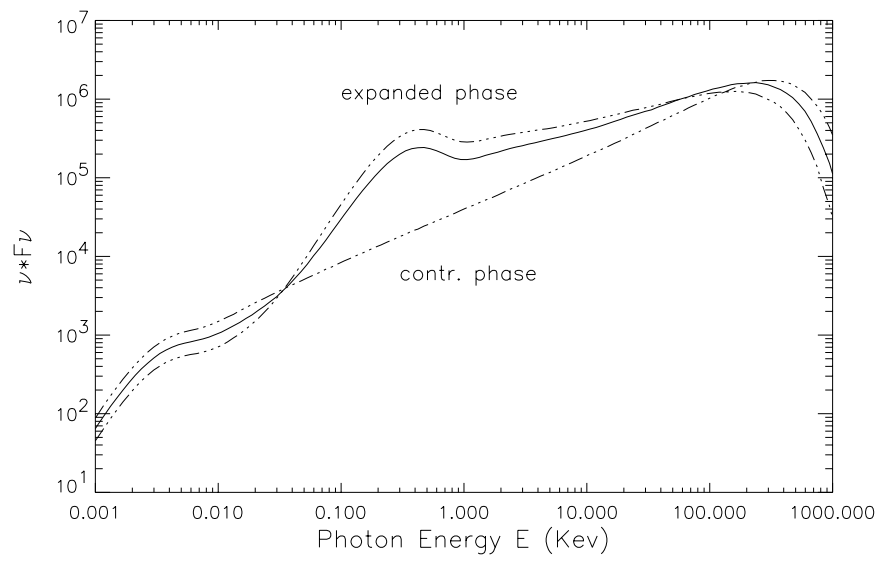

Fig. 5. Spectra predicted by the combined model of Fig. 3 for different phases of the oscillation. Solid line corresponds to the equilibrium position while dash-dotted lines correspond to maximum expansion and maximum contraction. $L_{\text {illum }} / L_{\mathrm{syn}}=230$ (see diamonds in Fig. 3).

optical/infrared wavelengths, if the synchrotron soft flux accounts for a large fraction of the total optical/infrared luminosity of the source. Implications from these conclusions are discussed in the next sections.

\section{Consequences/Predictions}

Radial oscillations of the ISAF excited by the interaction between an ISAF and a cool disk have a number of characteristics from which they can be identified and conclusions can be drawn about the accretion geometry. The most promising source of excitation turned out to be the interaction between ISAF and cool disk via proton illumination. For this mode of interaction, the model predicts the following.

i) The QPO frequency is predicted to be close to the Kepler frequency in the outer edge of the ISAF. For frequencies characteristic of the oscillations (around $1 \mathrm{~Hz}$ ), the ISAF must extend to $R_{\text {out }} \sim 100 R_{\mathrm{s}}$. This is comparable to the extent of the ISAF in the low/hard state in Cygnus X-1, as deduced by Gilfanov et al. (2000) from Fourier frequency-resolved spectroscopy.

ii) As shown in Sects. 5.2, 5.3 the excitation of the oscillations can be very fast, with $\eta / \omega \sim 1$. This implies that they are expected to show non-sinusoidal behavior and higher harmonics. Under these conditions our assumptions about almost sinusoidal oscillations are not valid any more. The main conclusions, however, are expected to stay qualitatively the same.

iii) There are cases with "hard" or finite amplitude instability, in which small amplitudes are damped but finite amplitudes can grow rapidly.

iv) If synchrotron flux accounts for part of the soft flux that is Comptonized, then infrared and/or visible modulations of the same frequency with the QPOs are predicted. The modulations will more likely be observed if synchrotron flux accounts for a large fraction of the total source emission in the relevant wavelengths.

v) Excitation of the oscillations occurs for overlap fraction of the ISAF and the cool disk less than about $7 \%$. If the observed QPOs are excited by the processes described here, there must be a rather sharp transition from the outer thin disk to the inner hot flow.

A final conclusion is that the QPOs are predicted to be soft, showing larger rms amplitudes in the soft X-ray bands than in the hard ones. This, at first sight, seems to conflict with the usual observational trend that QPOs are hard. There are, however, several observations of sources in the low/hard state where soft QPOs have been observed. During the decay after the 2000 outburst of XTE J1550-564 (Kalemci et al. 2001), or in the hard state of Cygnus X-1 (Rutledge et al. 1999) soft oscillations have been observed with frequencies around $1 \mathrm{~Hz}$. This suggests that there are several mechanisms contributing to QPO excitation, but makes clear that other mechanisms than those described here need to be explored.

\section{Summary and discussion}

The implications of any model that attempts to describe QPOs depend strongly on the assumed accretion geometry. In this work we have assumed that the accretion flow consists of a standard thin disk which is truncated in a radius $R_{\text {tr }}$ and a hot two-temperature ion supported flow (ISAF) inside this radius. Due to its tendency to spread outward viscously, the ISAF extends to a radius $R_{\text {out }}>R_{\text {tr }}$, resulting in partial overlap of the two flows.

We have focused on the frequency and the excitation/damping mechanisms of the fundamental mode of radial oscillations of the ISAF. The oscillatory frequency of this mode is close to the Kepler frequency in the outer edge of the ISAF. We find that the main contributor to the excitation of these oscillations is the variation of cooling rate of the ISAF in the different phases of the oscillation. When the ISAF cools more efficiently in the expanded phase than the contracted phase, an oscillation is excited.

We have assumed Compton cooling to be the dominant cooling physical process of the ISAF. Since the source of the Comptonized 'seed' photons depends on the still unknown details of the accretion geometry, we have considered three different physical mechanisms that have previously been proposed to account for the soft photon flux: i) synchrotron flux due to frozen in magnetic fields in the ISAF; ii) irradiation of the thin disk by X-rays from the ISAF (Haardt \& Maraschi 1993); and iii) proton illumination of the disk in the overlap region (Deufel \& Spruit 2000).

We have developed a Monte Carlo code to calculate the Compton scattering and to calculate the cooling of the ISAF, as well as the emergent X-ray spectra during the oscillations. We find that soft photons produced by synchrotron and by reprocessing of the hard flux act as damping mechanisms of the oscillations. On the other hand, proton illumination excites oscillations if the overlap fraction $f$ between the disk and the ISAF is small enough (of the order of $7 \%$ ). Also models that combine proton illumination with synchrotron or irradiation soft flux can produce (if some constraints are fulfilled) radial oscillations with amplitudes of the order of the overlap fraction, i.e. of the order $10 \%$. 
The oscillations are predicted to produce a QPO signature in the X-ray power spectrum that has more power in the soft $\mathrm{X}$-rays than in the hard X-rays (soft QPOs). This leads us to identify the low frequency QPOs observed in the decay of the 2000 outburst of XTE J1550-564 (Kalemci et al. 2001) and in the hard state of Cygnus X-1 (Rutledge et al. 1999) within the framework of our model.

Acknowledgements. We would like to thank the anonymous referee for useful suggestions that greatly improved the manuscript. Giannios acknowledges partial support from the E.C. Marie Curie Fellowship HPMTCT 2000-00132 and the Program "Heraklitos" of the Ministry of Education of Greece.

\section{Appendix A: The decay/excitation time scale}

The damping/growth rate $\eta$ of the amplitude of an oscillation can be defined as

$\eta=1 / 2 \frac{\langle\dot{W}\rangle}{\left\langle E_{\mathrm{osc}}\right\rangle}$,

where \langle\rangle expresses the average value of a quantity over a period, $E_{\text {osc }}$ is the oscillatory energy and $\dot{W}$ is the rate of energy gain of the oscillation. If $\langle\dot{W}\rangle$ is a negative number, then $\eta<0$ and corresponds to the damping rate of the oscillatory amplitude, while if $\langle\dot{W}\rangle$ is positive, then $\eta>0$ and corresponds to the excitation rate.

The total energy of the oscillation equals the kinetic energy of the oscillation when the medium crosses the equilibrium position. With Eq. (4), integrating over the volume of the ISAF we find

$E_{\mathrm{osc}}=\frac{2 \pi \tau m_{\mathrm{p}} a^{2} \omega^{2} R_{0}^{4}}{5 \sigma_{\mathrm{T}}}$.

For nearly sinusoidal oscillations, $\langle\dot{W}\rangle$ is given by the integral

$\langle\dot{W}\rangle=-\frac{1}{T} \int_{m} \mathrm{~d} m \int_{0}^{T}(\gamma-1) \frac{\Delta \rho}{\rho} \Delta \dot{q} \mathrm{~d} t$,

where $\Delta \dot{q}$ is the variation of the cooling rate per unit mass of the ISAF. In general $\Delta \dot{q}$ is function of the space coordinates and time. Taking the cooling rate constant over the volume of the medium (numerical checks show that this is rather realistic) and to vary sinusoidally with time, we have

$\Delta \dot{q}=\dot{q}_{\mathrm{amp}} \sin (\omega t+\phi)$.

With $\dot{q}_{\text {amp }}=\left(\dot{q}_{\text {exp }}-\dot{q}_{\text {contr }}\right) /\left(2 M_{\text {ISAF }}\right)$ being the amplitude of the oscillation of $\dot{q}$. Also $\dot{q}_{\text {exp }} / \dot{q}_{\text {contr }}$ is the cooling rate of the ISAF in maximum expansion/contraction and $M_{\mathrm{ISAF}}$ is the mass of the ISAF.

Expression (A.4) contains a phase $\phi$. For $-\pi / 2<\phi<\pi / 2$, the oscillation is excited, while for $\pi / 2<\phi<3 / 2 \pi$, the oscillation is damped. We will focus on the extreme cases $\phi=0$ and $\phi=\pi$. (The first corresponds to $\dot{q}_{\text {amp }}>0$ and the second to $\left.\dot{q}_{\text {amp }}<0\right)$.

Integrating (A.4) over a period and over the mass of the ISAF we find

$\langle\dot{W}\rangle=3(\gamma-1) a \frac{\dot{q}_{\exp }-\dot{q}_{\mathrm{contr}}}{4}$.
So, from Eq. (A.1) we can estimate the damping/growth rate of the amplitude of the oscillation. Of more interest is the quantity $\eta / \omega$, where $\omega$ is the the frequency of the oscillation, taken to be the Keplerian frequency at the outer edge of the ISAF.

This yields

$\frac{\eta}{\omega}=\frac{15}{4} \frac{(\gamma-1)}{\tau} \frac{c}{\left(G M / R_{\text {out }}\right)^{1 / 2}} \frac{L_{\mathrm{x}}}{L_{\mathrm{Edd}}} \frac{\left(\dot{q}_{\mathrm{exp}}-\dot{q}_{\mathrm{contr}}\right) / \dot{q}_{\mathrm{eq}}}{a}$

where $L_{\mathrm{x}}$ is the X-ray luminosity of the source and $\dot{q}_{\mathrm{eq}}$ is the the cooling rate of the ISAF in its equilibrium position. In order to derive this equation we have also used the expression for the Eddington luminosity $L_{\mathrm{E}}=4 \pi G M m_{\mathrm{p}} c / \sigma_{T}$ and the fact that $L_{\mathrm{x}} \simeq \dot{q}_{\mathrm{eq}}$ (since inverse Compton is responsible for both the cooling of the ISAF and the X-ray luminosity of the source).

The last expression can also be written more conveniently as

$\frac{\eta}{\omega}=\frac{3.5}{\tau} r_{\text {out }}^{1 / 2} \frac{L_{\mathrm{x}}}{L_{\mathrm{E}}} \frac{\left(\dot{q}_{\mathrm{exp}}-\dot{q}_{\mathrm{contr}}\right)}{a \dot{q}_{\mathrm{eq}}}$.

Note that the growth rate of the oscillation is proportional to $Y=\left(\dot{q}_{\text {exp }}-\dot{q}_{\text {contr }}\right) /\left(a \dot{q}_{\text {eq }}\right)$.

\section{References}

Armitage, P. J. 1998, ApJ, 501, L189

Belloni, T., Psaltis, D., \& van der Klis, M. 2002, ApJ, 512, 392

Blaes, O. M., \& Hawley, J. F. 1988, ApJ, 326, 277

Brandenburg, A., Nordlund, A., Stein, R. F., \& Torkelsson, U. 1995, ApJ, 446, 741

Churazov, E., Gilfanov, M., \& Revnivtsev, M. 2001, MNRAS, 321, 759

Cox, J. P. 1980, Theory of stellar pulsation (Princeton Series in Astrophysics)

Deufel, B., \& Spruit, H. C. 2000, A\&A, 362, 1

Deufel, B., Dullemond, C. P., \& Spruit, H.C. 2001, A\&A, 377, 955

Deufel, B., Dullemond, C. P., \& Spruit, H. C. 2002, A\&A, 387, 907

Drury, L.O'C. 1980, MNRAS, 193, 337

Gilfanov, M., Churazov, E., \& Revnivtsev, M. 2000, MNRAS, 316, 923

Gilham, S. 1981, MNRAS, 195, 755

Haardt, F., \& Maraschi, L. 1993, ApJ, 413, 507

Homna, F., Matsumoto, R., \& Kato, S. 1992, PASJ, 44, 529

Ipser, J. R. 1996, ApJ, 458, 508

Ichimaru, S. 1977, ApJ, 214, 840

Kaisig, M. 1989, A\&A 218, 89

Kalemci, E., Tomsick, J. A., Rothschild, R. E., Pottschmidt, K., \& Kaaret P. 2001, ApJ, 563, 239

Kato, S. 1978, MNRAS, 185, 629

Kato, S. 1990, PASJ, 42, 99

Kley, W., Papaloizou, J. C. B., \& Lin, D. N. C. 1993, ApJ, 409, 739

Liu, B. F., Mineshige, S., Meyer, F., Meyer-Hofmeister, E., \& Kawaguchi, T. 2002, ApJ 5475, 117

Lyubarskii, Y. 1997, MNRAS, 292, 679

Meyer, F., Liu, B. F., \& Meyer-Hofmeister, E. 2000, A\&A, 361, 175

Meyer-Hofmeister, E., \& Meyer, F. 2001, A\&A, 361, 175

Narayan, R., \& Yi, I. 1994, ApJ, 428, L13

Narayan, R., \& Yi, I. 1995, ApJ, 452, 710

Narayan, R., Goldreich, P., \& Goodman, J. 1987, MNRAS, 228, 1

Nayakshin, S., Kazanas, D., \& Kallman, T. R. 2000, ApJ, 537, 833

Nowak, M., A. 2000, MNRAS, 318, 361

Nowak, M. A., \& Wagoner R. V. 1991, ApJ, 378, 656 
Nowak, M. A., \& Wagoner R. V. 1992, ApJ, 393, 697

Nowak, M. A., Vaughan, B. A., Wilms, J., Dove, J. B., \& Begelman, M. C. 1999 , ApJ, 510, 874

Nowak, M. A., Wilms, J., \& Dove, J. B., 2002, MNRAS, 332, 856

Papaloizou, J. C. B., \& Stanley, G. Q. G. 1986, MNRAS, 220, 593

Pottschmidt, K., Wilms, J., Nowak, M. A. 2003, A\&A, 407, 1039

Poutanen, J., \& Fabian, A. C. 1999, MNRAS, 306, L31

Pozdnyakov, L. A., Sobol, I. M., \& Sunyaev, R. A. 1983, Astrophys. \& Space Phys. Rev. 2, 189

Psaltis, D., Belloni, T., \& van der Klis, M. 1999, ApJ, 520, 262

Rees, M. J., Phinney, E. S., Begelman, M. C., \& Blandford, R. D. 1982, Nature, 295, 17

Rezzolla, L., Yoshida, S' I., Maccarone, T. J., \& Zanotti, O. 2003, MNRAS, 344, L37
Rutledge, R. E., Lewin, W. H., van der Klis, M., et al. 1999, ApJS, 124,265

Shakura, N. I., \& Sunyaev, R. A. 1973, A\&A, 24, 337

Shapiro, S. L., Lightman, A. P., \& Eardley, D. M. 1976, ApJ, 204, 187

Spitzer L. 1956, Physics of Fully Ionised Gases (New York: Wiley)

Spruit, H. C., \& Deufel, B. 2002, A\&A, 387, 918

Stepney, S. 1983, MNRAS, 202, 467

Swank, J. 2001, Astrop. Space Sci., 276(S1), 201

Titarchuk, L., Lapidus, I., \& Muslimov, A. 1998, ApJ, 499, 315

Unno, W., Osaki, Y., Ando, H., \& Shibahashi, H. 1979, Nonradial oscillations of stars (University of Tokyo press)

Wardziński G., \& Zdziarski A.A. 2000, MNRAS, 314, 183

Zdziarski A.A. 1998, MNRAS, 296, L51

Zdziarski A.A., Lubiński P., \& Smith D.A. 1999, MNRAS, 303, L11 